\title{
HI-observations of blue compact dwarf galaxies. II. ${ }^{\star}$
}

\author{
W. K. Huchtmeier ${ }^{1}$, A. Petrosian ${ }^{2}$, Gopal-Krishna ${ }^{3}$, and D. Kunth ${ }^{4}$ \\ 1 Max-Planck-Institut für Radioastronomie, Auf dem Hügel 69, 53121 Bonn, Germany \\ e-mail: huchtmeier@mpifr-bonn.mpg.de \\ 2 Byurakan Astrophysical Observatory and Isaac Newton Institute of Chile, Armenian Branch, Byurakan 378433, Armenia \\ e-mail: artptrs@yahoo.com \\ 3 NCRA-TIFR, Pune University Campus, Pune 411 007, India \\ e-mail: krishna@ncra.tifr.res.in \\ 4 Institut d'Astrophysique, 75014 Paris, France \\ e-mail: kunth@iap.fr
}

Received 29 May 2006 / Accepted 14 October 2006

ABSTRACT

\begin{abstract}
We present new results from HI observations of previously undetected 69 blue compact dwarf galaxies (BCDGs) of which 35 BCDGs have been detected (detection rate 51\%). Optical data (e.g., from NED) and other literature search are used to estimate the likelihood of confusion from other galaxies within the Effelsberg beam. We have combined 24 BCDG galaxies from this study and from Paper I (Huchtmeier et al. 2005) that are unlikely to suffer from confusion effects in combination with 19 other similar BCDGs taken from the literature to infer several characteristic properties of the BCDG population. The parameters which were used for the statistical analysis include linear diameter, axial ratio, blue luminosity, HI and total masses, HI and total mass-to-blue luminosity ratios, HI to total mass ratio, $\mathrm{HI}$ gas velocity dispersion, oxygen abundance, equivalent width of $\mathrm{H} \beta$ emission line and $B-V$ color. The analysis has revealed that: BCDGs with higher HI mass tend to have lower abundances of heavy elements and are more compact; BCDGs with lower blue luminosity have larger equivalent width of $\mathrm{H} \beta$ emission line and bluer $B-V$ colors, as well as a tendency towards lower abundances of heavy elements; BCDGs with larger linear size, with higher total mass and those with higher total and HI mass per unit blue luminosity, tend to have broader HI emission line (larger velocity dispersion); there is no obvious dependence of the star formation parameters on the total mass; almost 10\% of the BCDGs emit milli-Jansky level radio continuum near $1 \mathrm{GHz}$.
\end{abstract}

Key words. galaxies: general - galaxies: dwarf - galaxies: evolution - galaxies: abundances - galaxies: starburst radio lines: galaxies

\section{Introduction}

Blue Compact Dwarf Galaxies (BCDGs) represent the subset of low-luminosity galaxies undergoing strong and short-lived episode of star formation at the present time. Starting from the pioneering work of Searle \& Sargent (1972), this class of galaxies has attracted increasing attention. However, the origin and peculiar nature of their starbursts is still poorly understood. HI observations of BCDGs are therefore important, for not only do they provide estimate of the total mass, but also reveal their HI content which is the raw material for the star formation. Both these, when combined with parameters like absolute luminosity, oxygen abundance etc., can furnish useful clues. In the first article of this series we presented HI observations of 56 BCDGs with the 100-m Effelsberg radiotelescope (Huchtmeier et al. 2005, hereafter referred to as Paper I). Recently, the full catalogue of the Second Byurakan Sky Survey (SBS) has been published (Stepanian 2005), most of which lack HI observations or detections. In this paper, we present sensitive HI observations of a sample of 69 BCDGs assembled from the SBS catalogue for which HI observations (or detection) has not been reported in the literature. Details of the sample are given in Sect. 2. The following sections present the HI observations, the deduced HI parameters, and a discussion of the results which also makes use

* Figure 1 is only available in electronic form at http://www . aanda.org of the data presented in Paper I, as well as the variety of optical observations gathered from literature, including the Sloan Digital Sky Survey (SDSS), and NED.

\section{The sample of BCDGs}

The sample of 69 BCDGs was selected from the Second objective prism surveys of active and star forming galaxies, conducted at Byurakan Observatory (Armenia). Detailed information on the Second Byurakan Survey (SBS) (Markarian \& Stepanian 1983; and the following papers) can be found in Stepanian (2005) and Petrosian et al. (2006a).

The selection criteria employed for this BCDGs sample are:

- $M_{\mathrm{p}}$ fainter than -17-mag, taking $H_{0}=75 \mathrm{~km} \mathrm{~s}^{-1} \mathrm{Mpc}^{-1}$;

- compact structure and a clear absence of spiral arms, or an obvious irregular morphology, as confirmed by high resolution imaging;

- the presence of strong and narrow emission lines;

- no observation (or detection) of the $21 \mathrm{~cm} \mathrm{HI}$ emission line reported in the literature.

\section{HI-observations and basic results}

The HI observations were made using the 100-m radiotelescope, which has a half power beam width of $9.3^{\prime}$ at a wavelength of $21 \mathrm{~cm}$. The 1024-channel autocorrelator was split 
into four filter banks ( 256 channels each) using a bandwidth of $6.25 \mathrm{MHz}$ which yielded a resolution of $6 \mathrm{~km} \mathrm{~s}^{-1}$ or $10 \mathrm{~km} \mathrm{~s}^{-1}$ after Hanning smoothing. A typical observing time of $60 \mathrm{~min}$ per source yielded a rms noise of $\sim 4 \mathrm{mJy}$ (the system noise was $30 \mathrm{~K}$ ). Most of the observations were repeated in order to improve the signal-to-noise ratio and the reliability. An ONsource position was combined with an OFF-source position every $10 \mathrm{~min}$. This total power mode improves the baseline stability of the spectra. Frequent measurements of well known continuum sources were used to control the pointing and calibration of the telescope. Every two to three hours, a well known line source (e.g., dwarf galaxies) was observed as a system check. The toolbox software of the MPIfR was used for the data reduction. The observed spectra were corrected for moderately curved baselines only; this should not introduce additional errors in the estimated velocities and flux densities of the lines since the line profiles are quite narrow in all cases.

From the observed 69 BCDGs, 35 have been detected in HI. This detection rate of $\sim 51 \%$ is markedly lower than $\sim 79 \%$ found for our BCDG sample reported in Paper I (Huchtmeier et al. 2005). The difference probably owes to the $\sim 2$ times higher average redshift for the present sample $(0.0159 \pm 0.0104$ versus $0.0072 \pm 0.0040)$.

The narrow profiles in Fig. 1 correspond to dwarfish galaxies (i.e., $M_{B, T}^{0, i}$ fainter than -15). In some profiles we have cut out the local HI emission from our galaxy (in fact, the difference of the local emission between the ON and OFF positions - total power mode), as it was much stronger than the extragalactic emission.

Table 1 summarizes the observational data: the galaxy name in Col. 1; coordinates (J2000) in Col. 2, as used for the observations (more precise optical coordinates will be published by Petrosian et al. 2006a); the optical dimensions in arcsec corresponding to the blue surface brightness level at $25 \mathrm{mag} \operatorname{arcsec}^{-2}$ (Petrosian et al. 2006b) in Col. 3; the blue apparent magnitude (Stepanian 2005; Petrosian et al. 2006b) in Col. 4; the optical heliocenctric radial velocity (Stepanian 2005; Petrosian et al. 2006b) in Col. 5. The HI data follow, i.e., the measured HI flux (Col. 6), the observed peak flux of the line and its rms error in Col. 7 (for non detections only the rms noise is shown), the heliocentric radial velocity derived from the midpoint of the line at $50 \%$ of the peak and its error (Col. 8), and the linewidth at a level of $50 \%$ of the line peak and, whenever clearly above the noise, the $20 \%$ level (Col. 9; uncertain values are followed by a ":"), and comments (Col. 10) where "c" is for possible confusion and "Int" for interacting galaxy.

The derived global parameters for not confused objects from our sample of galaxies are presented in Table 2, the galaxy name in Col. 1, the optical heliocentric velocity (Table 1, Col. 5) has been reduced to the frame of the cosmic microwave background (using NED) $V_{3 \mathrm{Kbgd}}$ (Col. 2). We did not use the more accurate $\mathrm{HI}$ velocities because of the possibility of confusion in some cases. The distances in Col. 3 have been derived taking a Hubble constant $H_{0}=72 \mathrm{~km} \mathrm{~s}^{-1} \mathrm{Mpc}^{-1}$ (Freedman et al. 2001). Optical diameters in the $D_{25}$ system (Table 1, Col. 3) have been corrected for absorption and for inclination, where the inclination was derived from the axial ratio assuming an intrinsic axial ratio of 0.2 (e.g. Tully 1985, in view of the uncertainties in these inclinations we did not apply corrections for possible type dependance of the intrinsic axial ratio):

$\log a_{0}=\log a+0.09 A_{b}-0.2 \log (a / b)$,

where the foreground absorption in the blue $A_{b}$ is from Schlegel et al. (1998, as given in the NED).
The linear diameter $A_{0, i}[\mathrm{kpc}]$ follows in Col. 4, the absolute magnitude $M_{b, t}^{0, i}$ corrected for Galactic extinction (Schlegel et al. 1998) and internal absorption in Col. 5. The internal absorption in a galaxy in the $B$-band, $A_{i}$, depends on inclination and luminosity (Giovanelli et al. 1994; Tully et al. 1998; Verheijen 2001; Karachentsev et al. 1999):

$A_{i}=\left[1.6+2.8\left(\log V_{m}-2.2\right)\right] \times \log (a / b) ; V_{m} \geq 42.7 \mathrm{~km} \mathrm{~s}^{-1}$

$A_{i}=0-$ other

$A_{i}=0-\mathrm{E}, \mathrm{S} 0, \mathrm{dSph}$.

Here we use the following definition of $V_{m}$ :

The HI rotational velocity $V_{m}=W_{50} /(2 \sin i)$ corrected for inclination and turbulent motion (Tully \& Fouqué 1985) with isotropic non-circular motion parameter $\sigma_{z}=8 \mathrm{~km} \mathrm{~s}^{-1}$.

The total H I mass (Col. 6) has been calculated using

$M_{\mathrm{HI}}=2.355 \times 10^{5} D^{2} \int S_{v} \mathrm{~d} v$

where $D$ is the distance in Mpc and $\int S_{v} \mathrm{~d} v$ is the integrated HI-flux in $\mathrm{Jy} \mathrm{km} \mathrm{s}^{-1}$.

The total mass $M_{T}$ (Col. 7) has been derived from:

$M_{T}=$ const. $D a_{0} \Delta v_{0, i}^{2}$,

where $D$ is the distance in Mpc, $a_{0}$ the corrected optical diameter in arcmin, const. $=33110$, and $\Delta v_{0, i}$ the corrected edgeon linewidths at $50 \%$ of the peak flux (e.g. Karachentsev et al. 2004).

The HI mass-to-luminosity ratio $M_{\mathrm{HI}} / L_{B}$, the mass-toluminosity ratio $M_{T} / L_{B}$, and the relative $\mathrm{HI}$ mass $M_{\mathrm{HI}} / M_{T}$ follow in Cols. 8 to 10 , respectively.

The radio continuum parameters of the detected BCDGs are based on the $\mathrm{NVSS}^{2}$ and/or the FIRST ${ }^{3}$ surveys at $1.4 \mathrm{GHz}$ (Sect. 4).

\section{Comments on individual galaxies}

The $21 \mathrm{~cm}$ receiver was tuned to the frequency corresponding to the optical radial velocity of each galaxy, hence the HI profile should appear in the center of its frame in Fig. 1. Profiles not centered to their frame might indicate confusion or blending with $\mathrm{HI}$ emission from other galaxies within the radio beam.

Around the position of each galaxy in our sample, we examinated a region of 9.3 arcmin radius (i.e. twice the half-power beam width of the Effelsberg telescope) using the Digital Sky Survey (DSS), as well as the velocity and other data provided in the NED. Based on this, if we failed to identify one or more likely sources of confusion to the observed HI profile, we accepted the HI profile to be genuinely associated with the BCDG.

SBS 0743+591B - the wide double-horned HI profile $(v=$ $6485 \mathrm{~km} \mathrm{~s}^{-1}$ ) is typical for a spiral galaxy and probably due to UGC $04020\left(v_{\text {opt }}=6295 \mathrm{~km} \mathrm{~s}^{-1}\right)$ at $1.5^{\prime} \mathrm{E}$, confused.

SBS 0926+606B - confused by SBS 0926+606A (1.5 arcmin south, optical velocity $v=4002 \mathrm{~km} \mathrm{~s}^{-1}$ ). The higher velocity peak of the HI profile agrees in velocity with galaxy B $\left(v=4090 \mathrm{~km} \mathrm{~s}^{-1}\right)$ and the low velocity extension matches with galaxy A (see Pustilnik et al. 2002).

SBS 1011+575 - in possible confusion with SDSS $\mathrm{J} 101419.34+571348.6\left(v=2970 \mathrm{~km} \mathrm{~s}^{-1}\right), 4^{\prime} \mathrm{S}, 11 \mathrm{~s} \mathrm{~W}$. The relatively wide observed profile might be due to contribution from this galaxy.

\footnotetext{
2 NRAO VLA Sky Survey at $20 \mathrm{~cm}$, Condon et al. (1998).

${ }^{3}$ Faint Images of the Radio Sky at $20 \mathrm{~cm}$, Becker et al. (1995).
} 
Table 1. Observational data.

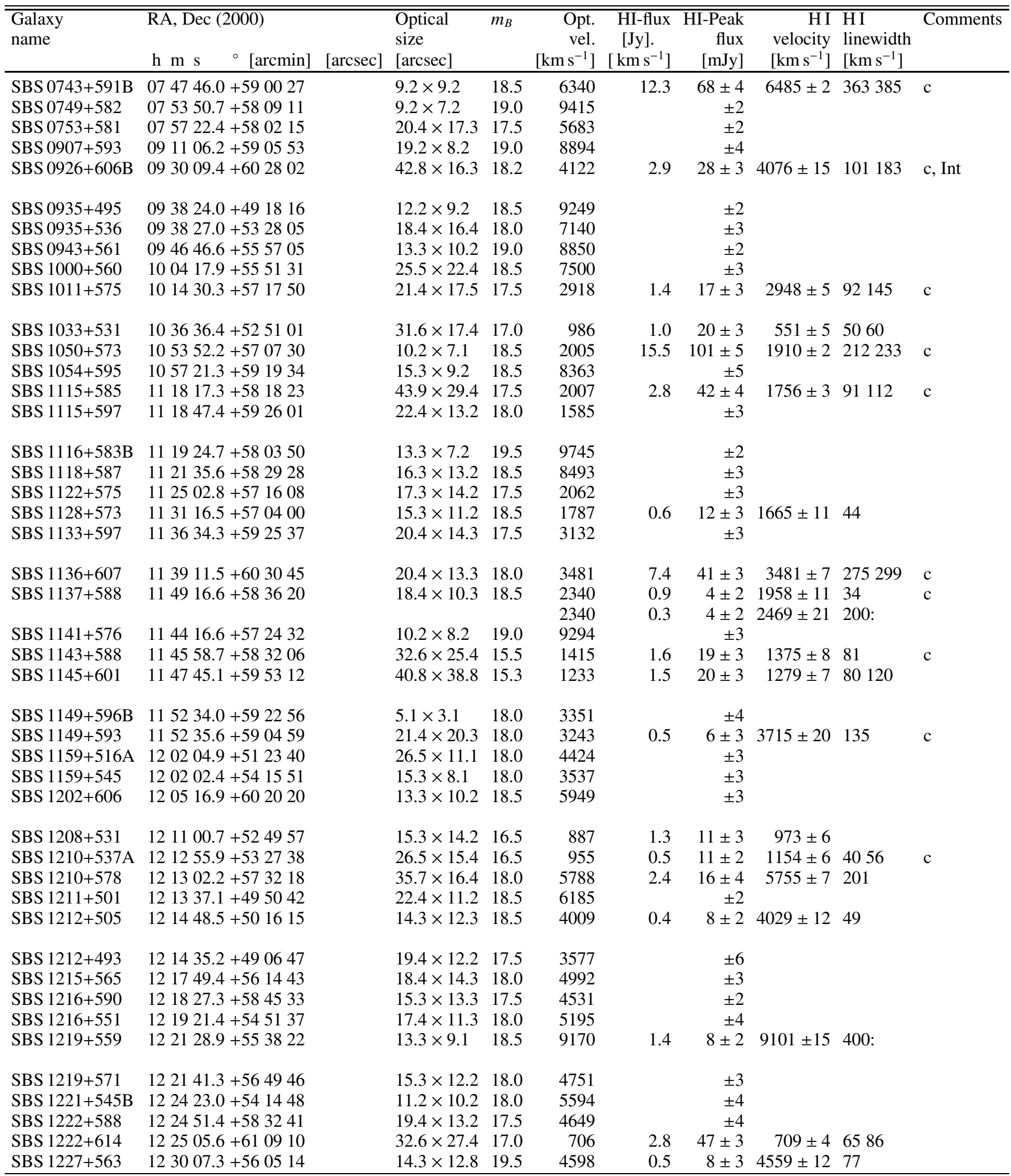

SBS 1033+531 - the brightest object within the Effelsberg beam. No confusing object in NED. A possible radio counterpart is seen on the NVSS image, with a peak flux of $\sim 2 \mathrm{mJy}$ at 1.4 GHz. However, non detection in the FIRST survey implies that any emission on arcsecond scale is weaker than $0.5 \mathrm{mJy}$ at $1.4 \mathrm{GHz}$.

SBS 1050+573 - falls within $1^{\prime}$ of NGC $3440(v=$ $1904 \mathrm{~km} \mathrm{~s}^{-1}$ ). Radial velocity of the compact dwarf galaxy is swamped by the wide double-horned HI profile due to
NGC 3440. An extended radio source of size $\sim 1.5 \times 0.9$ arcmin (position angle $58 \mathrm{deg}$ ) and flux density of $8.4 \pm 1.3 \mathrm{mJy}$ at $1.4 \mathrm{GHz}$ (NVSS), is associated with this BCDG. It is peaked

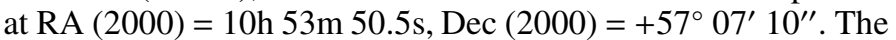
non detection in the FIRST survey implies that any arcsecond scale component is weaker than $0.5 \mathrm{mJy}$ at $1.4 \mathrm{GHz}$.

SBS 1115+585 - no HI emission detected at the optical velocity of this BCDG $\left(2007 \mathrm{~km} \mathrm{~s}^{-1}\right)$, the observed HI-profile is due to $\operatorname{UGC} 06304\left(v=1762 \mathrm{~km} \mathrm{~s}^{-1}\right)$. 
Table 1. continued.

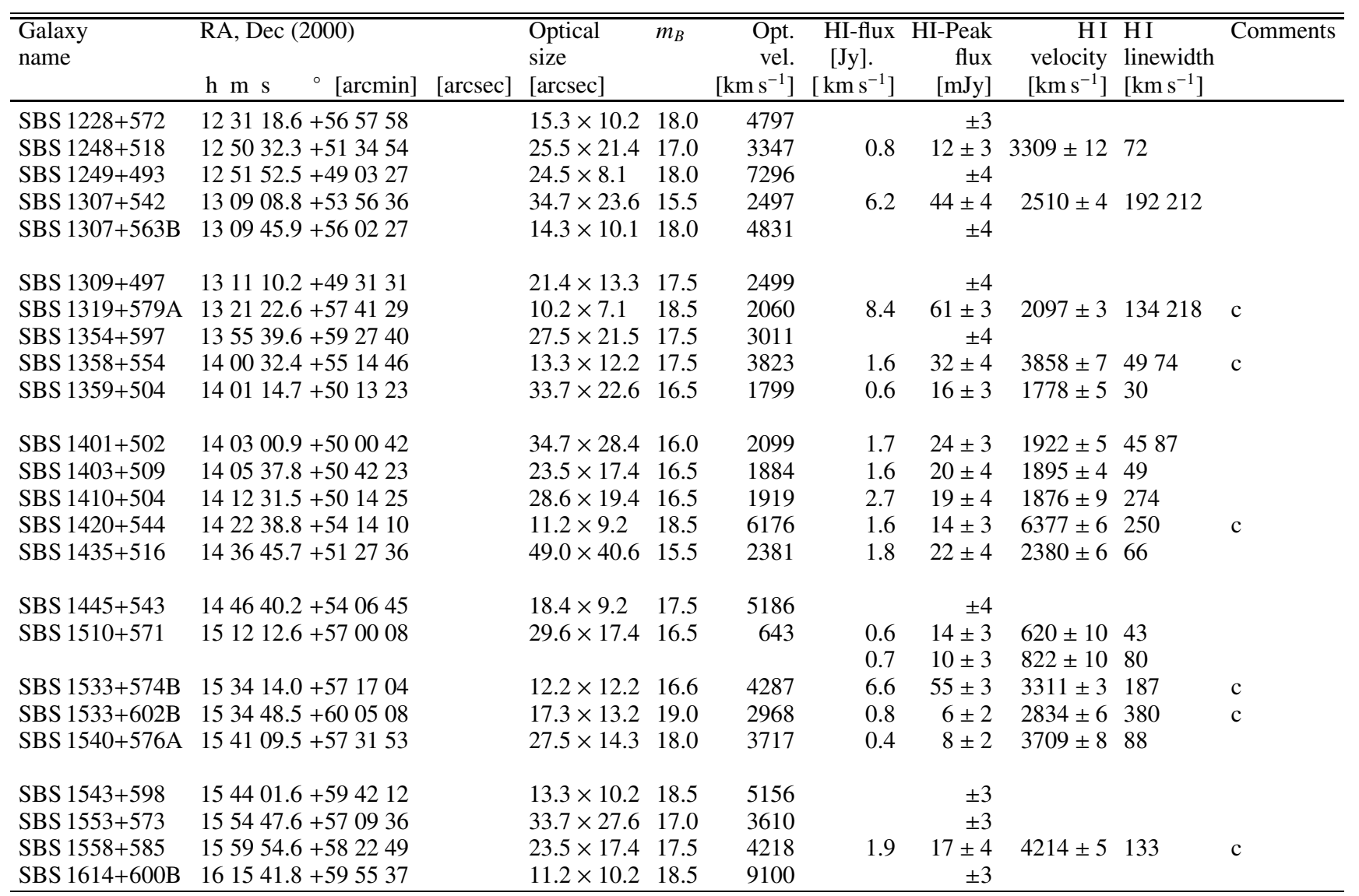

SBS 1128+573 - the observed HI profile $(v=1665 \pm$ $\left.11 \mathrm{~km} \mathrm{~s}^{-1}\right)$ is in marginal agreement with the optical velocity $\left(v=1787 \pm 36 \mathrm{~km} \mathrm{~s}^{-1}\right)$. No obvious confusing object found in NED.

SBS $1133+597$ - the FIRST image shows a point-like radio counterpart with flux density of $1.3 \pm 0.2 \mathrm{mJy}$ at $1.4 \mathrm{GHz}$, centered at RA $(2000)=11 \mathrm{~h} 36 \mathrm{~m} 34.9 \mathrm{~s}$, Dec $(2000)=+59^{\circ} 25^{\prime} 33^{\prime \prime}$.

SBS 1136+607 - the double-horned wide HI profile $(v=$ $3481 \mathrm{~km} \mathrm{~s}^{-1}$ ) is typical for a spiral galaxy, probably due to UGC $06619\left(v=3465 \mathrm{~km} \mathrm{~s}^{-1}\right), 3^{\prime} \mathrm{NE}$.

SBS 1137+588 - the weak HI emission at $v=2480 \mathrm{~km} \mathrm{~s}^{-1}$ ) is in fair agreement with the optical velocity $\left(v=2340 \mathrm{~km} \mathrm{~s}^{-1}\right)$. A possible HI feature seen at $v=1958 \mathrm{~km} \mathrm{~s}^{-1}$ could be due SBS $1137+589\left(v=2032 \mathrm{~km} \mathrm{~s}^{-1}\right) 2^{\prime} \mathrm{E}, 2.2^{\prime} \mathrm{N}$.

SBS 1143+588 - in possible confusion with the fainter HII galaxy SDSS J114603.39+583621.9 $\left(v=1414 \mathrm{~km} \mathrm{~s}^{-1}\right)$ situated $0.6^{\prime} \mathrm{E}$ and $4.2^{\prime} \mathrm{N}$. FIRST map shows a point-like image of $3.6 \pm$ $0.2 \mathrm{mJy}$ at $1.4 \mathrm{GHz}$.

SBS $1145+601$ - the HI velocity $\left(1279 \mathrm{~km} \mathrm{~s}^{-1}\right)$ is compatible with the optical velocity $\left(1232 \mathrm{~km} \mathrm{~s}^{-1}\right)$.

A faint radio counterpart is seen on the NVSS image, with a peak flux of $2 \pm 0.4 \mathrm{mJy}$ at $1.4 \mathrm{GHz}$, centred at

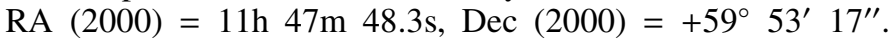
However, non detection in the FIRST survey implies that any arcsecond scale emission is weaker than $0.5 \mathrm{mJy}$ at $1.4 \mathrm{GHz}$.

SBS 1149+593 - no HI detection at the optical velocity.

SBS 1208+531 - no obvious confusing object in NED. Optical velocity $\left(887 \pm 37 \mathrm{~km} \mathrm{~s}^{-1}\right)$ within the combined errors is compatible with the HI velocity $\left(973 \pm 6 \mathrm{~km} \mathrm{~s}^{-1}\right)$.
SBS1210+537A - velocity of the HI profile $(v=$ $1154 \mathrm{~km} \mathrm{~s}^{-1}$ ) does not correspond to the optical velocity (955 $\left.\mathrm{km} \mathrm{s}^{-1} \mathrm{NED}\right)$. In confusion with SBS $1210+537 \mathrm{~B}$ at $0.7^{\prime}$ separation.

SBS 1210+578 - the HI velocity $\left(5755 \mathrm{~km} \mathrm{~s}^{-1}\right)$ is in agreement with the optical velocity $\left(5788 \mathrm{~km} \mathrm{~s}^{-1}\right)$. This SBS-galaxy is elongated. The broad HI-line is atypical of a dwarf galaxy.

SBS 1212+505 - the HI velocity $\left(4029 \mathrm{~km} \mathrm{~s}^{-1}\right)$ is in agreement with the optical velocity $\left(4009 \mathrm{~km} \mathrm{~s}^{-1}\right)$.

SBS 1219+559 - the weak double-peaked profile is too wide for a BCDG object. SBS $1219+559\left(v=9240 \mathrm{~km} \mathrm{~s}^{-1}\right)$ probably is confused by SBS $1219+558\left(v=9233 \mathrm{~km} \mathrm{~s}^{-1}\right)$ at $6^{\prime}$. The corresponding $\mathrm{HI}$ emisson could be the higher velocity peak. There is no optical counterpart in NED (with matching radial velocity) for the lower velocity HI peak. Due to these uncertainties this galaxy has not been included in Table 2 .

SBS 1222+614 - the HI velocity $\left(709 \mathrm{~km} \mathrm{~s}^{-1}\right)$ agrees well with the optical velocity $\left(706 \mathrm{~km} \mathrm{~s}^{-1}\right)$.

A faint point-like radio counterpart with $\sim 1 \mathrm{mJy}$ at $1.4 \mathrm{GHz}$ is seen on the FIRST image.

SBS $1227+563$ - the HI velocity $\left(4559 \mathrm{~km} \mathrm{~s}^{-1}\right)$ is in agreement with the optical velocity $\left(4598 \mathrm{~km} \mathrm{~s}^{-1}\right)$.

SBS 1248+518 - the HI velocity $\left(3309 \mathrm{~km} \mathrm{~s}^{-1}\right)$ is in agreement with the optical velocity $\left(3347 \mathrm{~km} \mathrm{~s}^{-1}\right)$. There is no sign for possible confusion within the radio beam.

SBS 1307+542 - the HI velocity $\left(2510 \mathrm{~km} \mathrm{~s}^{-1}\right)$ is in agreement with the optical velocity $\left(2458 \mathrm{~km} \mathrm{~s}^{-1}\right)$ of this galaxy but also of UGC $08231\left(v=2470 \mathrm{~km} \mathrm{~s}^{-1}\right)$ at $9^{\prime} \mathrm{NW}$. However, situated at $9^{\prime}$ from the SBS galaxy the contribution of UGC 08231 to the observed HI emission would only be of the order of $1 \mathrm{mJy}$, i.e. less than the noise of the profile. 
Table 2. Derived parameters for the detected galaxies

\begin{tabular}{rrrrrrrrrrr}
\hline \hline $\begin{array}{r}\text { Galaxy } \\
\text { name }\end{array}$ & $V_{3 \text { Kbgd }}$ & $\begin{array}{r}\text { Dist. } \\
D\end{array}$ & $\begin{array}{r}\text { Diam. } \\
A_{0, i}\end{array}$ & $\begin{array}{r}\text { Abs. } \\
\text { mag. }\end{array}$ & $\begin{array}{r}\text { HI } \\
\text { mass }\end{array}$ & $\begin{array}{r}\text { Total } \\
\text { mass }\end{array}$ & $M_{\mathrm{HI}} / L_{B}$ & $M_{T} / L_{B}$ & $M_{\mathrm{HI}} / M_{T}$ & Comments \\
$(\mathrm{SBS})$ & {$\left[\mathrm{km} \mathrm{s}^{-1}\right]$} & $\mathrm{Mpc}$ & $\mathrm{kpc}$ & $M_{B}$ & {$\left[10^{8} M_{\odot}\right]$} & {$\left[10^{8} M_{\odot}\right]$} & {$\left[M_{\odot} / L_{\odot}\right]$} & {$\left[M_{\odot} / L_{\odot}\right]$} & {$\left[M_{\odot} / L_{\odot}\right]$} & \\
\hline $1033+531$ & 1172 & 16 & 1.3 & -13.60 & 0.6 & 2.2 & 0.9 & 3.2 & 0.3 & \\
$1128+573$ & 1827 & 25 & 4.6 & -13.36 & 0.9 & 1.9 & 2.1 & 4.5 & 9.5 & \\
$1137+588 \mathrm{~B}$ & 2621 & 36 & 3.1 & -13.98 & 0.9 & 56 & 1.1 & 64 & 0.02 & \\
$1145+601$ & 1422 & 20 & 5.7 & -15.78 & 1.4 & 83.4 & 0.3 & 15.5 & 0.02 & \\
$1208+531$ & 1152 & 16 & 2.2 & -13.22 & 0.8 & 17.9 & 0.7 & 16.6 & 0.04 & \\
& & & & & & & & & & \\
$1210+578$ & 5908 & 82 & 4.0 & -16.40 & 38 & 206 & 5.4 & 29.4 & 0.2 & \\
$1212+505$ & 4221 & 59 & 1.8 & -15.15 & 3.2 & 10.2 & 1.4 & 4.4 & 0.3 & \\
$1222+614$ & 838 & 12 & 5.3 & -12.87 & 0.9 & 7.9 & 2.5 & 22 & 0.11 & \\
$1227+563$ & 5715 & 79 & 5.8 & -14.99 & 7.4 & 0.9 & 4.6 & 5.8 & 0.8 & \\
$1248+518$ & 3482 & 48 & 5.9 & -16.26 & 4.4 & 31.9 & 0.7 & 5.3 & 0.1 & \\
& & & & & & & & & & \\
$1307+542$ & 2663 & 37 & 1.4 & -16.97 & 20 & 132 & 1.4 & 9.0 & 0.2 & \\
$1359+504$ & 1918 & 27 & 4.7 & -15.40 & 1.0 & 1.2 & 0.3 & 0.4 & 0.8 & \\
$1401+502$ & 2061 & 29 & 3.1 & -16.11 & 3.3 & 7.7 & 0.6 & 1.4 & 0.4 & \\
$1403+509$ & 2030 & 28 & 3.1 & -15.44 & 3.0 & 4.5 & 0.9 & 1.3 & 0.7 & \\
$1410+504$ & 2008 & 28 & 3.6 & -15.36 & 5.0 & 160 & 1.5 & 49 & 0.03 & N5520 \\
& & & & & & & & & & \\
$1410+504$ & 2118 & 29 & 3.8 & -15.36 & 0.8 & 3.4 & 0.22 & 0.9 & 0.24 & \\
$1435+516$ & 2488 & 35 & 8.0 & -17.01 & 5.1 & 107 & 0.41 & 8.7 & 0.05 & \\
$1510+571 \mathrm{~B}$ & 682 & 9.5 & 1.2 & -13.12 & 0.13 & 0.9 & 0.34 & 2.5 & 0.14 & \\
$1510+571$ & 884 & 12 & 1.6 & -13.12 & 0.25 & 5.0 & 0.40 & 8.0 & 0.05 & U9776 \\
$1540+576 \mathrm{~A}$ & 3747 & 52 & 6.2 & -15.42 & 2.6 & 21 & 0.9 & 7.6 & 0.12 & \\
\hline
\end{tabular}

A faint radio counterpart is seen on the NVSS image, with a peak flux of $1.8 \pm 0.4 \mathrm{mJy}$ at $1.4 \mathrm{GHz}$, centred at RA $(2000)=13 \mathrm{~h}$ $09 \mathrm{~m}$ 08.6s, Dec $(2000)=+53^{\circ} 56^{\prime} 21^{\prime \prime}$. However, non detection in the FIRST survey implies that any arcsecond scale component is weaker than $0.5 \mathrm{mJy}$ at $1.4 \mathrm{GHz}$.

SBS 1319+579A - this object is the SW compact object projected on an elongated galaxy. SBS $1319+579 B$ is the central condensation of this galaxy. The object " $\mathrm{C}$ " is isolated and was not included in the latest SBS catalog (Petrosian et al. 2002). Optical velocities of these three components fall between 1958 and $2060 \mathrm{~km} \mathrm{~s}^{-1}$ and they approach the velocity of the observed HI profile $\left(v=2096 \mathrm{~km} \mathrm{~s}^{-1}\right)$. NGC $5109\left(v=2131 \mathrm{~km} \mathrm{~s}^{-1}\right)$ at $3.8^{\prime} \mathrm{W}, 3^{\prime} \mathrm{S}$ also matches in the $\mathrm{HI}$ velocity. The asymmetric shape of the profile is another hint for confusion.

SBS 1358+554 - in possible confusion with the BCDG SBS $1358+554 \mathrm{E}$ at $0.6^{\prime}$. This object is not included in the latest SBS catalog (Stepanian 2005). The HI velocity agrees with the optical velocities of this galaxy pair which is also observed by Comte et al. (1999).

SBS 1359+504 - the velocity of the faint HI emission $\left(1778 \mathrm{~km} \mathrm{~s}^{-1}\right)$ agrees with the optical velocity $\left(1799 \mathrm{~km} \mathrm{~s}^{-1}\right)$.

SBS 1401+502 - object is located near the edge of a Schmidt plate, the DSS image covers only part of the field of interest, no obvious confusing objects in NED.

SBS 1403+509 - the optical velocity $\left(1884 \mathrm{~km} \mathrm{~s}^{-1}\right)$ is in agreement with the HI velocity $\left(1895 \mathrm{~km} \mathrm{~s}^{-1}\right)$. Only a minor confusion $(\leq 1 \mathrm{mJy})$ is expected from NGC $5480\left(v=1856 \mathrm{~km} \mathrm{~s}^{-1}\right)$ $8^{\prime} \mathrm{W}$, due to the strong tapering of the antenna pattern at this distance from the beam center.

SBS1410+504 - the asymmetric HI profile is a hint of confusion. Indeed, the velocity of the HI profile $\left(1876 \mathrm{~km} \mathrm{~s}^{-1}\right)$ is close to the optical velocity of NGC $5520\left(1846 \mathrm{~km} \mathrm{~s}^{-1}\right)$ at $7^{\prime} \mathrm{NW}$. A secondary peak on the HI profile at $v=1900 \mathrm{~km} \mathrm{~s}^{-1}$ corresponds to the optical velocity $\left(1919 \mathrm{~km} \mathrm{~s}^{-1}\right)$ of this BCDG. The HI-data in Table 1 correspond to this secondary profile alone.
The NVSS shows a faint radio counterpart, with a peak flux of $1.4 \pm 0.4 \mathrm{mJy}$ at $1.4 \mathrm{GHz}$, centred at RA (2000) =

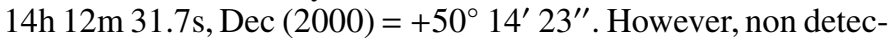
tion in the FIRST survey implies that any arcsecond scale emission is below $0.5 \mathrm{mJy}$ at $1.4 \mathrm{GHz}$.

SBS 1420+544 - the velocity of the wide HI profile $\left(6377 \mathrm{~km} \mathrm{~s}^{-1}\right)$ does not coincide with the optical velocity $\left(6176 \mathrm{~km} \mathrm{~s}^{-1}\right)$. Moreover, the broad line is not typical for BCDGs. However, there is no obvious sign of a confusing object in NED.

SBS 1435+516 - HI and optical velocities coincide (2380 and $\left.2381 \mathrm{~km} \mathrm{~s}^{-1}\right)$. NGC $5707\left(v=2212 \mathrm{~km} \mathrm{~s}^{-1}\right)$ at $9.3^{\prime}$ will not contribute more than 1 or $2 \mathrm{mJy}$ to the observed HI profile.

SBS 1510+571 - the HI profile shows two well separated features $\left(v=620\right.$ and $\left.822 \mathrm{~km} \mathrm{~s}^{-1}\right)$ which correspond to the optical velocities of this BCDG $\left(v=643 \mathrm{~km} \mathrm{~s}^{-1}\right)$ centered to the frame in Fig. 1 and to UGC $09776\left(v=833 \mathrm{~km} \mathrm{~s}^{-1}\right)$ at $7.7^{\prime}$.

SBS 1533+574B - pairs with the galaxy SBS 1533+574 A. The velocity centroid of the broad HI profile $\left(3311 \mathrm{~km} \mathrm{~s}^{-1}\right)$ agrees better with the optical velocity of companion $\mathrm{A}(v=$ $3348 \mathrm{~km} \mathrm{~s}^{-1}$ ) (Petrosian et al. 2002). Possible confusion also with CGCG 297-017 $\left(v=3282 \mathrm{~km} \mathrm{~s}^{-1}\right)$ at 5.2'. No HI emission observed at the radial velocity of the galaxy.

A point-like faint radio counterpart $(\sim 0.5 \mathrm{mJy}$ at $1.4 \mathrm{GHz})$ is seen on the FIRST map.

SBS 1533+602B - this broad albeit noisy HI line profile is not typical for BCDGs. Moreover, the optical $\left(v=2968 \mathrm{~km} \mathrm{~s}^{-1}\right)$ and $\mathrm{HI}$ velocities $\left(2834 \mathrm{~km} \mathrm{~s}^{-1}\right)$ are only in marginal agreement.

SBS 1540+576A - the radial velocity of the observed narrow $\mathrm{HI}$ line $\left(3709 \mathrm{~km} \mathrm{~s}^{-1}\right.$ ) agrees well with the optical velocity $\left(3717 \mathrm{~km} \mathrm{~s}^{-1}\right)$.

SBS $1553+573$ - a possible radio counterpart $(\leq 0.5 \mathrm{mJy}$ at $1.4 \mathrm{GHz}$ ) is seen near the detection limit of the FIRST survey.

SBS 1558+585 - good agreement between optical $\left(4218 \mathrm{~km} \mathrm{~s}^{-1}\right)$ and $\mathrm{HI}$ velocity $\left(4214 \mathrm{~km} \mathrm{~s}^{-1}\right)$. A brighter 
object, SBS $1559+585$ with $v=4229 \mathrm{~km} \mathrm{~s}^{-1}$ ) at $2.2^{\prime}$ E probably confuses the HI profile (see Paper I).

Thus, from the afore-mentioned discussions, we find that the HI detections in the present sample of 69 BCDGs, 19 BCDGs show no evidence of confusion in their observed HI profiles. Secondly, milli-Jansky level radio emission at $1.4 \mathrm{GHz}$ is found to be associated with the BCDGs SBS 1033+531, SBS 1050+573, SBS 1133+597, SBS 1143+588, SBS 1145+601, SBS 1222+614, SBS $1307+542$, SBS $1410+504$, SBS $1533+574 B$ and possibly SBS $1553+573$ (total 9 BCDGs).

\section{Discussion}

Mainly during the last two decades, several evolutionary schemes have been developed to unify different morphological classes of dwarf galaxies and to understand their star formation history (e.g. Skillman \& Bender 1995; Ferrara \& Tolstoy 2000). In all these models, the amount of HI gas is the key ingredient, being the fuel driving the star formation. When HI data are used in conjunction with other parameters of these galaxies, mainly, the metallicity and the stellar population markers, this places constraints on the star formation history (e.g., Lee et al. 2002). Such studies are sensitively dependent on the size of the sample of galaxies and the choice of parameters employed. Combining the HI detected BCDGs from Paper I and the present observations, we count 79 objects for the further statistical study. Out of these total $79 \mathrm{HI}$ detected BCDGs, we have assessed 48 BCDGs to be essentially free from confusion effects (Sect. 4; Paper I). Spectral and photometric information for 24 of these BCDGs could be gathered from the Sloan Digital Sky Survey (SDSS) Data Release 4(DR4) and for a few BCDGs from other literature (Isotov \& Thuan 1999; Stepanian et al. 2002; Lee et al. 2004; Petrosian et al. 2006a,b). Further, in order to improve the statistics, we searched the literature to find more BCDGs with published HI, spectral and photometric data, while ensuring that these BCDGs also conform to the defining criteria adopted for our sample (Sect. 2). Our literature search yielded the requisite data for 19 BCDGs, mostly from the articles by Lee et al. (2002, 2004), Salzer et al. (2002, 2005), Thuan \& Martin (1981), Petrosian et al. (2006a) and from NED. This brings the total available sample to $43 \mathrm{BCDGs}$ (including $24 \mathrm{BCDGs}$ from our sample, as described above). In all, 12 parameters were selected for statistical study, these are grouped in three categories:

(a) integral parameters, namely, linear diameter: $A_{0, i}$ in $\mathrm{Kpc}$; logarithm of axial ratio: $\log b / a$ and $\operatorname{logarithm}$ of blue luminosity: $\log L_{B}$; $\log$ arithm of total mass: $\log M_{\text {tot }}$ in solar units; logarithm of the total mass to blue luminosity ratio: $\log M_{\text {tot }} / L_{B}$

(b) HI related parameters, namely, logarithm of HI mass: $\log M_{\mathrm{HI}}$ in solar units; logarithm of HI mass-to-blue luminosity ratio: $\log M_{\mathrm{HI}} / L_{B}$; logarithm of $\mathrm{HI}$ mass-to-total mass ratio: $\log M_{\mathrm{HI}} / M_{\mathrm{tot}}$ and the $\mathrm{HI}$ linewidth at $50 \%$ of the peak: $W_{50}$ in $\mathrm{km} \mathrm{s}^{-1}$; and

(c) parameters related to star formation: oxygen abundance log $\mathrm{O} / \mathrm{H}$; equivalent width of $\mathrm{H} \beta$ emission line: $E W-\mathrm{H} \beta$ in Angstroms and $B-V$ color. For the objects with SDSS spectra, oxygen abundances are computed through the direct electron temperature $\left(T_{\mathrm{e}}\right)$ method, or estimated using the ratio [OIII] $5007 / \mathrm{H} \beta$, or [NII]6583/ $\mathrm{H} \alpha$, or both emission line ratios (Salzer et al. 2005; Shi et al. 2005). SDSS DR4 $(g-r)$ colors were transformed to $(B-V)$ colors according to the equation $B-V=0.62(g-r)+0.15$ of Jester et al. (2005). The number of BCDGs taken from our samples and from the literature, for which we could determine all the 12 parameters, is 43 . Table 3 summarizes this information.

We now subject the extensive data available for the 43 BCDGs to the so called, Multivariate Factor Analysis (MFA) (Harman 1967; Afifi \& Azen 1979). MFA is a statistical test for detecting correlations among a set of $\mathrm{m}$ initial variables measured on $\mathrm{n}$ objects, through a reduced number $(p<m)$ of linearly independent factors $F_{1}, F_{2}, \ldots F_{p}$ that presumably account for the correlation. The MFA gives a description, or explanation for the interdependence of a set of variables in terms of the $F_{i}$ factors without regard to the observed variability. A detailed description of the MFA method can be found in Harman (1967) and Afifi \& Azen (1979). This method has been used successfully in astronomy by several authors (e.g., Petrosian \& Turatto 1992; Patat et al. 1994; Petrosian et al. 2003). For the present analysis, the parameters in Table 3 have been selected as the initial variables. In order to represent each initial variable with the smaller number of common factors and for a simpler interpretation of the results, we apply the Varimax orthogonal rotation (Kaiser 1958) to the three factors that minimizes the number of common factors for each initial variable. Table 4 shows the factor loadings, i.e. the correlation coefficients between the initial variables and the $F_{i}$ factors. Accumulated dispersion by the first three Varimax rotated factors is $70 \%$. Adopting $r \sim 0.6$ as correlation threshold, the first rotated factor, $F_{1}$, which accounts for about $17 \%$ of the common dispersion, is dominated by neutral gas $\log M_{\mathrm{HI}}$ and $\log M_{\mathrm{HI}} / M_{\text {tot }}$ parameters, oxygen abundance $(\log \mathrm{O} / \mathrm{H})$ and axial ratio $(a / b)$, with higher HI mass BCDGs showing lower abundances of heavy elements and being more compact. This result is in good agreement with Lee et al. (2002). Similar result also was obtained in the HI study of KISS low-luminosity galaxies which are mostly BCDGs, or related objects. Factor two, $F_{2}$, describing about $21 \%$ of the total variance, correlates optical luminosity of BCDGs with their star formation markers, $E W-\mathrm{H} \beta$ and $B-V$ color, with lower blue luminosity BCDGs having larger equivalent widths of $\mathrm{H} \beta$ emission line and bluer $(B-V)$ colors and with a correlation coefficient of 0.472 lower heavy element abundances $(\log \mathrm{O} / \mathrm{H}$ parameter). Similar result for different samples of BCDGs and related low-luminosity galaxies have been obtained in the past (e.g., Salzer et al. 2005; Shi et al. 2005; Lee et al. 2004; Kong et al. 2002; Lee et al. 2006). The third Factor, $F_{3}(32 \%)$, is the combination of the $A_{0, i}, \log M_{\mathrm{tot}}, \log M_{\mathrm{tot}} / L_{B}$, $\log M_{\mathrm{HI}} / L_{B}$ and $W_{50}$ parameters, BCDGs of larger linear size, higher total mass and $\mathrm{HI}$ mass per unit blue luminosity, having a larger velocity dispersion of the HI gas. The correlation of HI velocity dispersion with integral parameters has also been noted in some recent studies (e.g. Lee et al. 2002) and the positive correlation between luminosity of BCDGs and their HI gas velocity dispersion is a reflection of the well known Tully-Fisher (1977) relation for this sample of dwarf galaxies. According to the theory underlying the MFA, the factors are orthogonal, hence the corresponding initial variables are independent. It means that for this sample of BCDGs there is no obvious relation between star forming parameters and total mass of these galaxies.

\section{Conclusions}

We have recorded a $51 \%$ detection rate in our Effelsberg $21 \mathrm{~cm}$ HI observations of a sample of 69 galaxies. For a statistical study of the properties of BCDGs we selected 12 different parameters which group their integral and HI properties, and parameters related to star formation. These data are available for 24 out 
Table 3. Objects and parameters for the statistical analysis.

\begin{tabular}{|c|c|c|c|c|c|c|c|c|c|c|c|c|}
\hline Galaxy & $\begin{array}{r}D_{0} \\
{[\mathrm{kpc}]}\end{array}$ & $\frac{b}{a}$ & $\begin{array}{r}\log \\
L_{B}\end{array}$ & $W_{50}$ & $\begin{array}{c}\log \\
M_{\mathrm{HI}}\end{array}$ & $\begin{array}{l}\log \\
\frac{M_{\mathrm{HI}}}{L_{B}}\end{array}$ & $\begin{array}{l}\log \\
M_{T}\end{array}$ & $\begin{array}{l}\log \\
\frac{M_{T}}{L}\end{array}$ & $\begin{array}{r}\log \\
\frac{M_{\mathrm{HI}}}{M_{T}}\end{array}$ & $\frac{\mathrm{O}}{\mathrm{H}}$ & $E W\left(\mathrm{H}_{\beta}\right)$ & $(B-V)$ \\
\hline Mrk 1416 & 4.3 & 0.517 & 8.31 & 58 & 9.00 & $\frac{L_{B}}{0.36}$ & 8.73 & 0.43 & $\frac{L_{T}}{-0.07}$ & 7.86 & 85.00 & 0.30 \\
\hline Mrk 1446 & 6.8 & 0.879 & 8.75 & 50 & 8.54 & -0.21 & 9.29 & 0.53 & -0.74 & 8.13 & 77.10 & 0.25 \\
\hline Mrk 1450 & 1.7 & 0.875 & 8.27 & 77 & 7.30 & -0.97 & 9.08 & 0.82 & -2.00 & 7.98 & 123.60 & 0.14 \\
\hline Mrk 1460 & 1.2 & 0.650 & 7.43 & 36 & 6.78 & -0.65 & 7.70 & 0.28 & -0.89 & 8.11 & 58.30 & 0.32 \\
\hline SBS $0943+543$ & 2.2 & 0.632 & 7.71 & 46 & 7.00 & -0.71 & 8.23 & 0.53 & -1.10 & 8.28 & 24.90 & 0.27 \\
\hline SBS $1006+578 \mathrm{~A}$ & 3.1 & 0.469 & 8.07 & 105 & 8.45 & 0.38 & 9.12 & 1.04 & -0.68 & 8.28 & 4.70 & 0.32 \\
\hline SBS $1054+504$ & 3.0 & 0.613 & 8.35 & 56 & 7.48 & -0.87 & 8.58 & 0.23 & -1.05 & 8.23 & 18.22 & 0.43 \\
\hline SBS $1114+587$ & 3.1 & 0.846 & 8.23 & 52 & 7.78 & -0.45 & 8.86 & 0.62 & -1.05 & 8.24 & 5.14 & 0.38 \\
\hline SBS $1118+578 B$ & 3.7 & 0.875 & 8.51 & 43 & 7.78 & -0.73 & 8.85 & 0.34 & -1.10 & 8.26 & 5.90 & 0.40 \\
\hline SBS $1128+573$ & 1.8 & 0.730 & 7.54 & 44 & 7.95 & 0.42 & 8.28 & 0.65 & -0.30 & 8.17 & 113.90 & 0.13 \\
\hline SBS $1205+557$ & 2.3 & 0.722 & 7.95 & 61 & 7.48 & -0.47 & 8.66 & 0.76 & -1.15 & 7.91 & 83.10 & 0.26 \\
\hline SBS $1208+531$ & 1.2 & 0.930 & 7.48 & 80 & 7.90 & 0.42 & 9.25 & 1.22 & -1.40 & 8.11 & 28.30 & 0.31 \\
\hline SBS $1211+540$ & 1.1 & 0.813 & 7.23 & 47 & 7.30 & 0.07 & 8.23 & 1.00 & -0.85 & 7.64 & 124.50 & 0.11 \\
\hline SBS $1212+505$ & 4.0 & 0.860 & 8.25 & 49 & 8.51 & 0.25 & 9.01 & 0.64 & -0.52 & 7.95 & 101.40 & 0.26 \\
\hline SBS $1222+614$ & 1.8 & 0.840 & 7.34 & 65 & 7.95 & 0.61 & 8.90 & 1.34 & 0.96 & 8.02 & 87.20 & 0.15 \\
\hline SBS $1227+563$ & 5.3 & 0.790 & 8.19 & 49 & 8.87 & 0.68 & 8.97 & 0.76 & -0.10 & 8.03 & 24.40 & 0.32 \\
\hline SBS $1248+518$ & 5.8 & 0.840 & 8.70 & 72 & 8.64 & -0.05 & 9.50 & 0.72 & 1.00 & 8.19 & 4.70 & 0.36 \\
\hline SBS $1423+517$ & 4.3 & 0.613 & 8.35 & 79 & 8.26 & -0.10 & 9.11 & 0.77 & 0.85 & 8.26 & 131.90 & 0.31 \\
\hline SBS $1428+457$ & 6.1 & 0.919 & 8.87 & 119 & 9.01 & 0.14 & 10.25 & 1.39 & 1.22 & 8.26 & 85.10 & 0.27 \\
\hline SBS $1430+526$ & 7.5 & 0.829 & 8.83 & 72 & 9.03 & 0.20 & 9.57 & 0.73 & -0.54 & 8.03 & 42.69 & 0.27 \\
\hline SBS $1435+516$ & 8.0 & 0.830 & 9.00 & 112 & 8.71 & -0.29 & 10.03 & 0.94 & 1.30 & 8.40 & 2.70 & 0.39 \\
\hline SBS $1453+526$ & 5.6 & 0.500 & 8.67 & 199 & 8.49 & -0.18 & 10.95 & 1.25 & 1.52 & 8.08 & 71.28 & 0.34 \\
\hline SBS $1510+571 B$ & 1.6 & 0.590 & 7.44 & 43 & 7.40 & -0.04 & 8.70 & 0.90 & 1.30 & 8.26 & 6.00 & 0.35 \\
\hline SBS $1540+576 A$ & 6.2 & 0.520 & 8.36 & 88 & 8.41 & 0.05 & 9.32 & 0.88 & -0.92 & 8.24 & 4. 90 & 0.32 \\
\hline \multicolumn{13}{|l|}{$\begin{array}{l}\text { Data from the } \\
\text { literature }\end{array}$} \\
\hline Mrk 0067 & 1.6 & 0.690 & 7.80 & 62 & 7.42 & -0.38 & 8.86 & 1.06 & -1.44 & 8.21 & 82.60 & 0.09 \\
\hline Mrk 0475 & 1.2 & 0.680 & 7.67 & 40 & 8.27 & 0.60 & 8.36 & 0.69 & -0.09 & 7.93 & 138.90 & 0.02 \\
\hline Mrk 0487 & 1.1 & 0.920 & 7.96 & 75 & 7.45 & -0.51 & 8.87 & 0.91 & -1.42 & 8.06 & 164.94 & 0.24 \\
\hline Mrk 0600 & 3.0 & 0.440 & 8.55 & 73 & 8.54 & -0.01 & 9.28 & 0.73 & -0.74 & 7.83 & 31.30 & 0.30 \\
\hline Mrk 0996 & 3.6 & 0.760 & 8.91 & 83 & 7.18 & -1.73 & 9.47 & 0.56 & -2.29 & 7.98 & 129.00 & 0.27 \\
\hline Mrk 1308 & 3.4 & 1.000 & 8.91 & 31 & 6.65 & -2.26 & 8.59 & -0.33 & -1.94 & 8.67 & 12.00 & 0.39 \\
\hline IZw18 & 0.9 & 0.670 & 7.72 & 53 & 7.84 & 0.12 & 8.49 & 0.77 & -0.65 & 7.18 & 100.00 & -0.06 \\
\hline IIZw40 & 1.7 & 0.390 & 7.76 & 99 & 8.38 & 0.62 & 9.30 & 1.54 & -0.92 & 8.09 & 283.00 & 0.10 \\
\hline IIZw70 & 4.3 & 0.350 & 8.68 & 69 & 8.57 & -0.11 & 9.39 & 0.71 & -0.82 & 8.06 & 49.00 & 0.27 \\
\hline VIIZw403 & 1.6 & 0.570 & 8.03 & 43 & 8.25 & 0.22 & 8.58 & 0.55 & -0.33 & 7.69 & 155.30 & 0.38 \\
\hline KISSR0096 & 6.1 & 0.650 & 8.92 & 55 & 9.25 & 0.34 & 9.34 & 0.42 & -0.09 & 7.91 & 32.00 & 0.43 \\
\hline KISSR0133 & 6.8 & 0.460 & 8.99 & 40 & 7.96 & -1.03 & 9.11 & 0.12 & -1.15 & 8.14 & 8.50 & 0.54 \\
\hline KISSR0286 & 3.8 & 0.890 & 8.81 & 211 & 8.79 & -0.02 & 10.31 & 1.49 & -1.51 & 8.17 & 50.00 & 0.56 \\
\hline KISSR0310 & 4.5 & 0.730 & 8.98 & 168 & 8.68 & -0.31 & 10.18 & 1.20 & -1.50 & 7.88 & 136.00 & 0.60 \\
\hline KISSR0396 & 4.4 & 0.400 & 8.19 & 47 & 8.05 & -0.13 & 9.06 & 0.87 & -1.00 & 7.92 & 48.94 & 0.34 \\
\hline KISSR0471 & 19.4 & 0.349 & 8.98 & 191 & 9.32 & 0.32 & 10.92 & 1.94 & 1.60 & 7.49 & 39.03 & 0.15 \\
\hline KISSR0666 & 9.7 & 0.620 & 8.54 & 58 & 8.61 & 0.06 & 9.59 & 1.04 & 0.98 & 7.77 & 116.40 & 0.41 \\
\hline KISSR0785 & 7.3 & 0.829 & 8.75 & 124 & 9.01 & 0.25 & 10.12 & 1.37 & 1.11 & 7.95 & 22.80 & 0.43 \\
\hline KISSR1021 & 2.4 & 0.750 & 8.15 & 110 & 8.21 & 0.06 & 9.53 & 1.38 & 1.32 & 8.49 & 11.52 & 0.32 \\
\hline
\end{tabular}

of the non-confused BCDGs reported in the present work and Paper I. These data in combination with those for 19 other similar BCDGs from the literature are used to infer several characteristics of the BCDG population of 43 galaxies. Some statistically significant trends found for our sample are:

1. BCDGs with greater HI mass have lower abundances of heavy elements and are more compact. Such a correlation first given by Chamaraux (1977) is in accord with the idea that gas-rich low luminosity galaxies have converted a smaller fraction of their neutral gas into stars (StaveleySmith et al. 1992).

2. BCDGs having lower blue luminosity show larger equivalent widths of $\mathrm{H} \beta$ emission line and bluer $(B-V)$ colors (albeit, the correlation coefficient is only 0.472 for the case of heavy element abundances).
3. Higher HI velocity dispersion is positively correlated with linear size, total mass, HI mass, as well as the HI mass per unit blue luminosity.

These three properties point to the same underlying fact i.e. that gas-rich low luminosity galaxies tend to be metalpoor systems (e.g. Lequeux et al. 1979; Kinman \& Davidson 1981; Skillman et al. 1989). Indeed the metallicity - luminosity relationship found by Richer \& McCall (1995) holds in our sample although with a large scatter as we expect in small systems undergoing transient bursts of star formation and hence evolving rapidly with time. To a first approximation metallicity anticorrelates with the gas mass fraction as expected from closed box models (cf. Lequeux et al. 1979; Kinman \& Davidson 1981; Pagel 1997) but this can not be the whole explanation (Matteucci \& Chiosi 1983). Note that not all dwarf galaxies comply with the correlation 
Table 4. Varimax rotated factor matrix.

\begin{tabular}{rrrr}
\hline \hline & $F 1$ & $F 2$ & $F 3$ \\
\hline$A_{0, i}$ & 0.450 & 0.441 & 0.627 \\
$b / a$ & -0.601 & 0.073 & -0.096 \\
$\log L_{B}$ & 0.081 & 0.752 & 0.444 \\
$W_{50}$ & -0.098 & 0.105 & 0.909 \\
$\log M_{\mathrm{HI}}$ & 0.607 & 0.323 & 0.524 \\
$\log M_{\mathrm{HI}} / L_{B}$ & 0.400 & -0.073 & 0.607 \\
$\log M_{\text {tot }}$ & 0.040 & 0.351 & 0.899 \\
$\log M_{\text {tot }} / L_{B}$ & 0.044 & -0.350 & 0.817 \\
$\log M_{\mathrm{HI}} / M_{\text {tot }}$ & 0.738 & -0.045 & -0.473 \\
$12+\log \mathrm{O} / \mathrm{H}$ & -0.601 & 0.472 & -0.180 \\
$E W(\mathrm{H} \beta)$ & 0.034 & -0.708 & 0.100 \\
$(B-V)$ & -0.176 & 0.807 & 0.077 \\
Acc. Variance $(\%)$ & 17 & 38 & 70 \\
\hline & & &
\end{tabular}

between metal abundance and luminosity. Indeed many of them appear overluminous for their abundance (Rönnback \& Bergvall 1995; Kunth \& Östlin 2000). If the underlying correlation is between mass and metallicity this is expected since most BCDGs have lower $M / L$ ratios (see Kennicutt \& Skillman 2001, for a thorough discussion of the problem).

4. No dependence is evident between the total galactic mass and the star formation indicators.

All these results are consistent with previous results for different samples of low-luminosity dwarf galaxies. In particular no more galaxies with extreme properties such as very low metallicity have been found (Kunth \& Östlin 2000).

5. At least 7, but probably 9 (i.e., 13\%) BCDGs in the present sample show evidence for milli-Jansky level radio continuum at $1.4 \mathrm{GHz}$. The radio counterpart of the BCDG $1050+573$ is clearly resolved (size $\sim 1.5 \times 0.9$ arcmin). If the present BCDG sample is combined with the BCDG sample reported in Paper I, then the mJy level radio continuum is detected in at least 12 of the total 125 BCDGs (i.e., nearly $10 \%$ ).

Acknowledgements. Based on observations with the 100-m radio telescope of the MPIfR (Max-Planck-Institut für Radioastronomie) at Effelsberg. We have made extensive use of the NASA/IPAC Extragalactic Database (NED, which is operated by the Jet Propulsion Laboratory, Caltech, under contract with the National Aeronautics and Space Administration), and the Digitized Sky Survey (DSS-1) produced at the Space Telescope Science Institute under US Government grant NAG W-2166. G.K. thanks the MPIfR (Germany) and A.P. thanks the IAP (France) for hospitality during a period when part of this work was done. The authors thank an anonymous referee for useful comments.

\section{References}

Afifi, A. A., \& Azen, S. P. 1979, Statistical Analisis: A Computer Oriented Approach (Chicago: Academic Press)

Becker, R. H., White, R. L., \& Delfand D. J. 1995, ApJ, 450, 559 (FIRST)
Chamaraux, P., 1977, A\&A, 60, 67

Comte, G., Petrosian, A. R., Ohanian, G. A., \& Stepanian, J. A. 1999, Astrophysics, 42, 149

Condon, J. J., Cotton, W. D., Greisen, E. W., et al. 1998, AJ, 115, 1693 (NVSS)

Ferrara, A., \& Tolstoy, E. 2000, MNRAS, 313, 291

Freedman, W. L., Madore, B. F., Gibson, B. K., et al. 2001, ApJ, 553, 47

Giovanelli, R., Haynes, M. P., Salzer, J. J., et al. 1994, AJ, 107, 2036

Huchtmeier, W. K., Krishna, G., \& Petrosian, A. 2005, A\&A, 434, 887 (Paper I)

Hurman, H. H. 1967, Modern Factor Analisis, 2nd Ed. (Chicago: Univ. of Chicago Press)

Isotov, Y. I., \& Thuan, T. X. 1999, ApJ, 511, 639

Jester, S., Schnerder, D. P., Richards, G. J., et al. 2005, AJ, 130, 873

Kaiser, H. F. 1958, Psychomet., 23, 187

Karachentsev, I. D., Makarov, D. I., \& Huchtmeier, W. K. 1999, A\&AS, 139, 97

Karachentsev, I. D., Karachentseva, V. E., Huchtmeier, W. K., \& Makarov, D. I., 2004, AJ, 127, 2031 [KKHM]

Kennicutt, R. C., \& Skillman, E. D. 2001, AJ, 121, 1461

Kinman, T. D., \& Davidson, K. 1981, ApJ, 243, 127

Kong, X., Cheng, F. Z., Weiss, A., \& Charlot S. 2002, A\&A, 396, 503

Kunth, D., \& Östlin, G. 2000, A\&ARv, 10, 1

Lee, J. C., Salzer, J. J., Impey, C., Thuan, T. X., \& Gronwall, C. 2002, AJ, 124, 3088

Lee, J. C., Salzer, J. J., \& Melbourne, J. 2004, ApJ, 616, 752

Lee, H., Skillman, E. D., Cannon, J. M., et al. 2006 [arXiv: astro-ph/0605036]

Lequeux, J., Peimbert, M., Rayo, J.-F., Serrano, A., \& Torres-Peimbert, S. 1979, A\&A, 80, 155

Markarian, B. E. 1967, Astrophys., 3, 34

Markarian, B. E., \& Stepanian, J. A. 1983, Astrophys., 19, 354

Markarian, B. E., Lipovetskii, V. A., Stepanian, J. A., Erastova, L. K., \& Shapovalova, A. I. 1989, Soobshch. Spets. Astrofiz. Obs., 62, 5

Matteucci, F., \& Chiosi, C. 1983, AAP, 123, 121

Mazzarella, J. M., \& Balzano, V. A. 1986, ApJS, 62, 751

Pagel, B. E. J. 1997, Nucleosynthesis and Chemical Evolution of Galaxies (Cambridge: University Press)

Patat, F., Barbon, R., Cappellaro, E., \& Turatto, M. 1994, A\&A, 282, 731

Petrosian, A., \& Turatto, M. 1992, A\&A, 261, 433

Petrosian, A., McLean, B., Allen, R. J., et al. 2002, AJ, 123, 2280

Petrosian, A., Allen, R. J., Leitherer, C., et al. 2003, AJ, 125, 86

Petrosian, A. R., Navasardyan, H., Cappellaro, E., et al. M. 2005, AJ, 129, 1369

Petrosian, A. R., McLean, B., Allen R. J., \& MacKenty, J. 2006a, ApJS, submitted

Petrosian, A. R., McLean, B., Stepanian, J. A., et al. 2006b, in preparation Pustilnik, S. A., Martin J.-M., Huchtmeier, W. K., et al. 2002, A\&A, 389, 405

Richer, M. G., \& McCall, M. L. 1995, ApJ, 445, 642

Rönnback, J., \& Bergvall, N. 1995, AAP, 302, 353

Salzer, J. J., Rosenberg, J. L., Weisstein, E. W., Mazzarella, J. M., \& Bothun G. D., 2002, AJ, 124, 191

Salzer, J. J., Lee, J. C., Melbourne, J., et al. 2005, ApJ, 624, 661

Schlegel, D. J., Finkbeiner, D. P., \& Davies, M. 1998, ApJ, 500, 525

Searle, L., \& Sargent, W. 1972, ApJ, 173, 25

Shi, F., Kong, X., Li, C., \& Chag, F. Z. 2005, A\&A, 437, 849

Skillman, E. D., \& Bender R. 1995, Rev. Mex. Astron. Astrofis. Ser. Conf., 3, 25

Skillman, E.D., Kennicutt, R. C., \& Hodge, P. W. 1989, ApJ, 347, 875

Staveley-Smith, L., Davies R. D., \& Kinman, T. D. 1992, MNRAS, 258, 334

Stepanian, J. 2005, Rev. Mex. Astron. Astrofis., 41, 155

Stepanian, J. A., Chavushian, V. H., Carrasco, L., et al. 2002, AJ, 124, 1283

Thuan, T. X., \& Martin, G. E. 1981, ApJ, 247, 823

Tully, R. B. 1985, Nearby Galaxies Catalog (Cambridge: Cambridge University Press)

Tully, R. B., \& Fisher J. R., 1977, A\&A, 54, 661

Tully, R. B., Pierce, J., Huang, J. S., et al. 1998, AJ, 115, 2264

Verheijen, M. A. W. 2001, ApJ, 563, 694 


\section{Online Material}


W. K. Huchtmeier et al.: HI-observations of blue compact dwarf galaxies. II., Online Material p 2
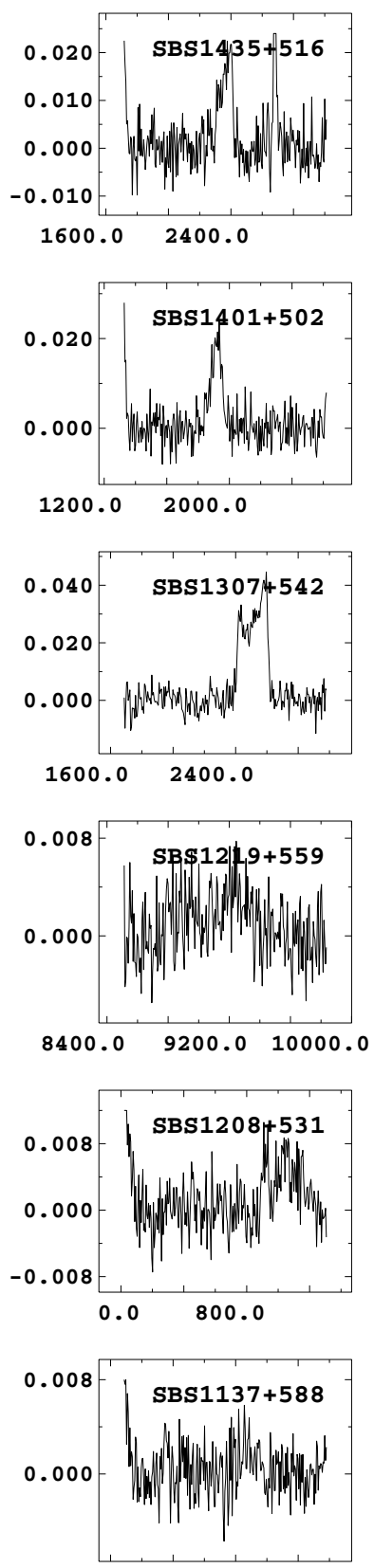

$1600.0 \quad 2400.0$
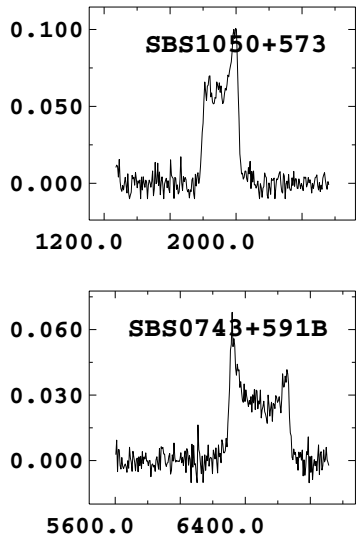
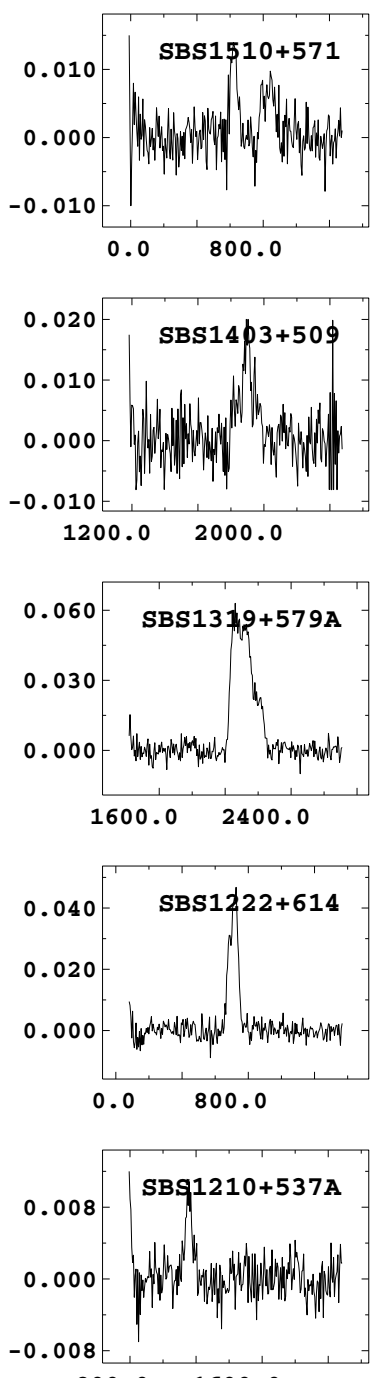

$800.0 \quad 1600.0$
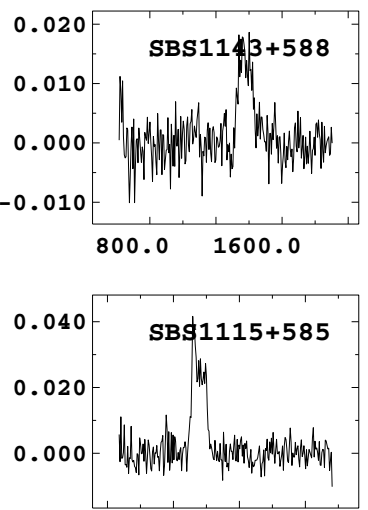

$1200.0 \quad 2000.0$

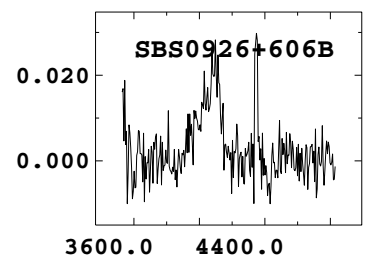

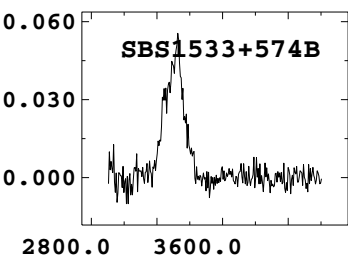

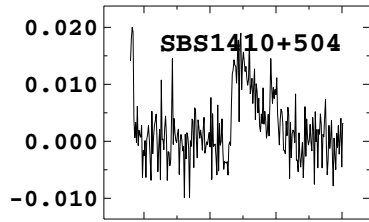

$$
1200.0 \quad 2000.0
$$
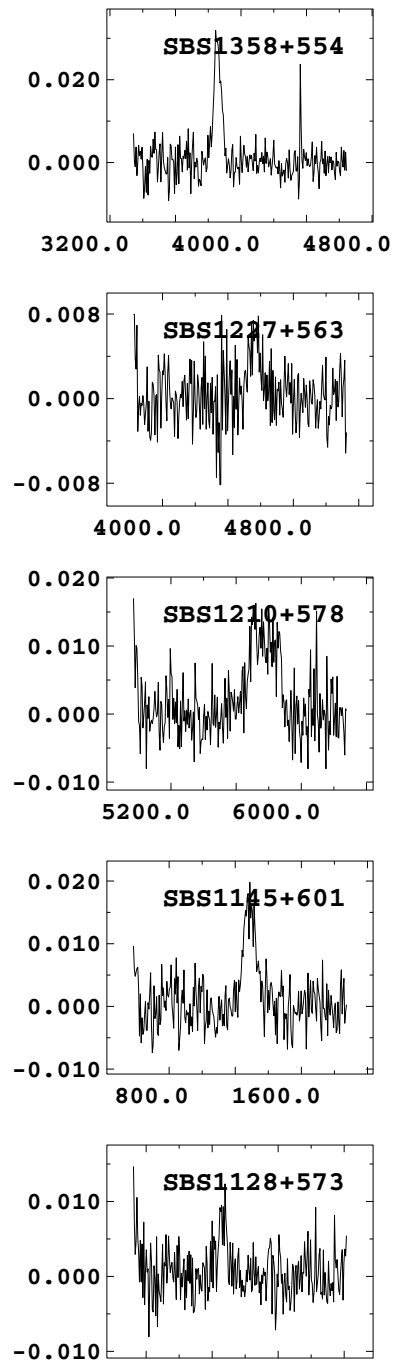

$$
1200.02000 .0
$$

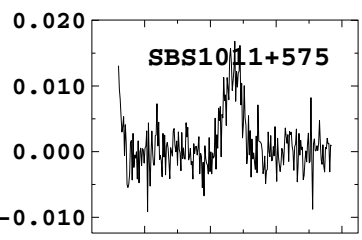

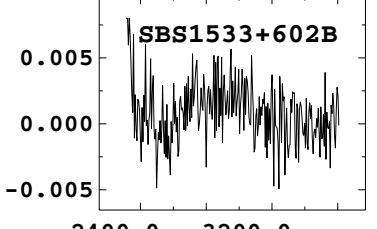

$2400.0 \quad 3200.0$

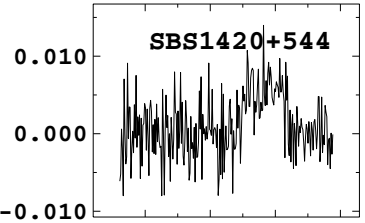

$5600.0 \quad 6400.0$

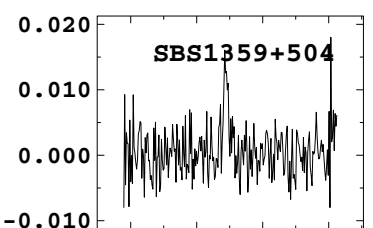

$1200.0 \quad 2000.0$
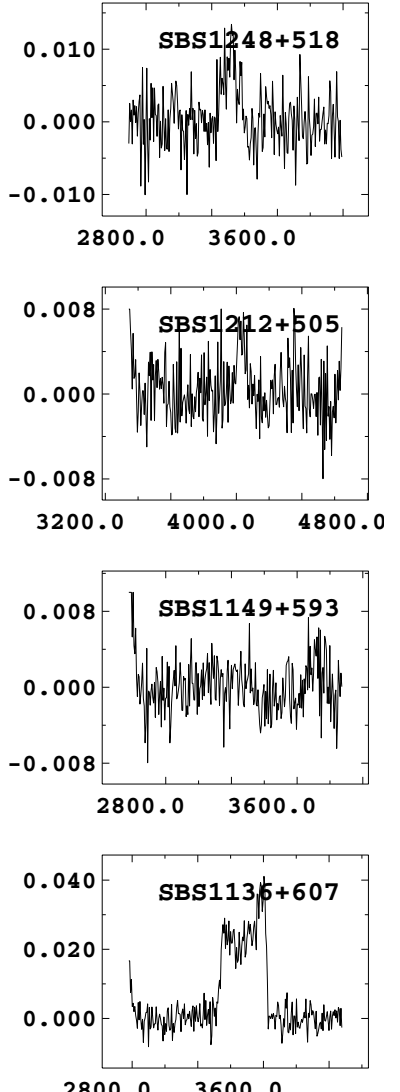

$2800.0 \quad 3600.0$

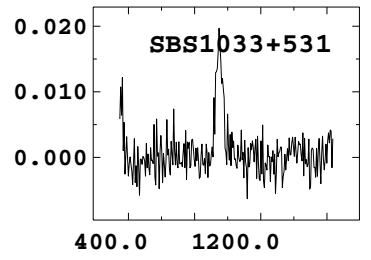

Fig. 1. HI profiles of the 34 BCDGs detected in the present Effelsberg observations, with a pencil beam of half-power beamwidth of 9.3 arcmin at $21 \mathrm{~cm}$. All these galaxies have been detected for the first time. Profiles are arranged in an increasing order of right ascension, starting at the bottom left corner (see Table 1). The axes are labelled in Jansky (Jy) and in heliocentric radial velocity in $\mathrm{km} \mathrm{s}^{-1}$. An "X" following the galaxy name indicates possible confusion by another galaxy within the telescope beam (Sect. 4). 
W. K. Huchtmeier et al.: HI-observations of blue compact dwarf galaxies. II., Online Material p 3
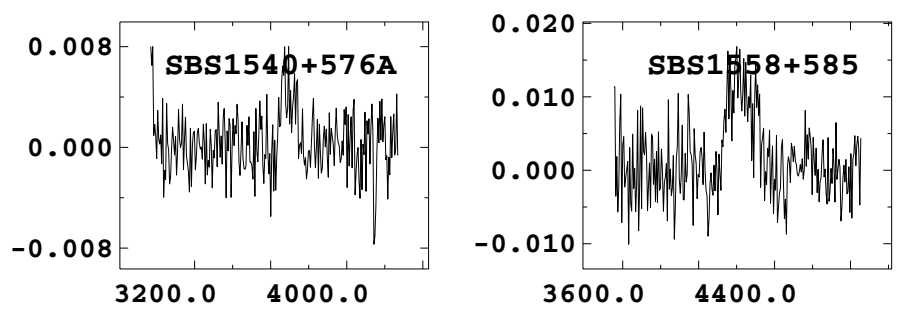

Fig. 1. continued. 